\title{
GENERALIZATIONS OF EULER NUMBERS AND POLYNOMIALS
}

\section{QIU-MING LUO, FENG QI, and LOKENATH DEBNATH}

\author{
Received 5 November 2002
}

\begin{abstract}
The concepts of Euler numbers and Euler polynomials are generalized and some basic properties are investigated.
\end{abstract}

2000 Mathematics Subject Classification: 11B68, 33E20.

1. Introduction. It is well known that the Euler numbers and polynomials can be defined by the following definitions.

DEFINITION 1.1 (see [1]). The Euler numbers $E_{k}$ are defined by the following expansion:

$$
\operatorname{sech} t=\frac{2 e^{t}}{e^{2 t}+1}=\sum_{k=0}^{\infty} \frac{E_{k}}{k !} t^{k}, \quad|t| \leq \pi .
$$

In [6, page 5], the Euler numbers are defined by

$$
\frac{2 e^{t / 2}}{e^{t}+1}=\operatorname{sech} \frac{t}{2}=\sum_{n=0}^{\infty} \frac{(-1)^{n} E_{n}}{(2 n) !}\left(\frac{t}{2}\right)^{2 n}, \quad|t| \leq \pi .
$$

DEFINITION 1.2 (see $[1,6]$ ). The Euler polynomials $E_{k}(x)$ for $x \in \mathbb{R}$ are defined by

$$
\frac{2 e^{x t}}{e^{t}+1}=\sum_{k=0}^{\infty} \frac{E_{k}(x)}{k !} t^{k}, \quad|t|<\pi .
$$

Let $\mathbb{N}$ denote the set of all positive integers. It can also be shown that the polynomials $E_{i}(t), i \in \mathbb{N}$, are uniquely determined by the following two properties:

$$
\begin{gathered}
E_{i}^{\prime}(t)=i E_{i-1}(t), \quad E_{0}(t)=1, \\
E_{i}(t+1)+E_{i}(t)=2 t^{i} .
\end{gathered}
$$

Euler polynomials are related to the Bernoulli numbers. For information about Bernoulli numbers and polynomials, we refer to [1, 2, 3, 5, 6].

In this note, we give some generalizations of the concepts of Euler numbers and Euler polynomials and research their basic properties. In fact, motivations 
and ideas of this note and other articles, see, for example, [2, 3, 4], originate essentially from [5].

2. Generalizations of Euler numbers and polynomials. In this section, we give two definitions, the generalized Euler number and the generalized Euler polynomial, which generalize the concepts of Euler number and Euler polynomial.

DEFINITION 2.1. For positive numbers $a, b$, and $c$, the generalized Euler numbers $E_{k}(a, b, c)$ are defined by

$$
\frac{2 c^{t}}{b^{2 t}+a^{2 t}}=\sum_{k=0}^{\infty} \frac{E_{k}(a, b, c)}{k !} t^{k} .
$$

DEFINITION 2.2. For any given positive numbers $a, b$, and $c$ and $x \in \mathbb{R}$, the generalized Euler polynomials $E_{k}(x ; a, b, c)$ are defined by

$$
\frac{2 c^{x t}}{b^{t}+a^{t}}=\sum_{k=0}^{\infty} \frac{E_{k}(x ; a, b, c)}{k !} t^{k} .
$$

Taking $a=1$ and $b=c=e$, then Definitions 1.1 and 1.2 can be deduced from Definitions 2.1 and 2.2, respectively. Thus, Definitions 2.1 and 2.2 generalize the concepts of Euler numbers and polynomials.

3. Some properties of the generalized Euler numbers. In this section, we study some basic properties of the generalized Euler numbers defined in Definition 2.1.

THEOREM 3.1. For positive numbers $a, b$, and $c$ and real number $x \in \mathbb{R}$,

$$
\begin{gathered}
E_{0}(a, b, c)=1, \quad E_{k}(1, e, e)=E_{k}, \quad E_{k}\left(1, e^{1 / 2}, e^{x}\right)=E_{k}(x), \\
E_{k}(a, b, c)=2^{k}(\ln b-\ln a)^{k} E_{k}\left(\frac{\ln c-2 \ln a}{2(\ln b-\ln a)}\right), \\
E_{k}(a, b, c)=\sum_{j=0}^{k}\left(\begin{array}{l}
k \\
j
\end{array}\right)(\ln b-\ln a)^{j}(\ln c-\ln a-\ln b)^{k-j} E_{j} .
\end{gathered}
$$

Proof. The formulas in (3.1) follow from Definitions 1.1, 1.2, and 2.1 easily. By Definitions 1.2 and 2.1 and direct computation, we have

$$
\begin{aligned}
\frac{2 c^{t}}{b^{2 t}+a^{2 t}} & =\frac{2 \exp ((\ln c-2 \ln a) / 2(\ln b-\ln a) \cdot 2 t(\ln b-\ln a))}{\exp (2 t(\ln b-\ln a))+1} \\
& =\sum_{k=0}^{\infty} 2^{k}(\ln b-\ln a)^{k} E_{k}\left(\frac{\ln c-2 \ln a}{2(\ln b-\ln a)}\right) \frac{t^{k}}{k !}
\end{aligned}
$$

Then, formula (3.2) follows. 
Substituting $E_{k}(x)=\sum_{j=0}^{k} 2^{-j}\left(\begin{array}{l}k \\ j\end{array}\right)(x-1 / 2)^{k-j} E_{j}$ into the formula (3.2) yields formula (3.3). The proof of the classical result for $E_{k}(x)$ follows from the more general proof that will be given for (4.1).

THEOREM 3.2. For $k \in \mathbb{N}$,

$$
\begin{gathered}
E_{k}(a, b, c)=-\frac{1}{2} \sum_{j=0}^{k-1}\left(\begin{array}{l}
k \\
j
\end{array}\right)\left[(2 \ln b-\ln c)^{k-j}+(2 \ln a-\ln c)^{k-j}\right] E_{j}(a, b, c), \\
E_{k}(a, b, c)=E_{k}(b, a, c), \\
E_{k}\left(a^{\alpha}, b^{\alpha}, c^{\alpha}\right)=\alpha^{k} E_{k}(a, b, c) .
\end{gathered}
$$

Proof. By Definition 2.1, direct calculation yields

$$
\begin{aligned}
1 & =\frac{1}{2}\left[\left(\frac{b^{2}}{c}\right)^{t}+\left(\frac{a^{2}}{c}\right)^{t}\right] \sum_{k=0}^{\infty} \frac{t^{k}}{k !} E_{k}(a, b, c) \\
& =\frac{1}{2} \sum_{k=0}^{\infty} \frac{t^{k}}{k !}\left[\left(\ln \frac{b^{2}}{c}\right)^{k}+\left(\ln \frac{a^{2}}{c}\right)^{k}\right] \sum_{k=0}^{\infty} \frac{t^{k}}{k !} E_{k}(a, b, c) \\
& =\frac{1}{2} \sum_{k=0}^{\infty}\left(\sum_{j=0}^{k}\left(\begin{array}{l}
k \\
j
\end{array}\right)\left[\left(\ln \frac{b^{2}}{c}\right)^{k-j}+\left(\ln \frac{a^{2}}{c}\right)^{k-j}\right] E_{j}(a, b, c)\right) \frac{t^{k}}{k !} .
\end{aligned}
$$

Equating coefficients of $t^{k}$ in (3.8) gives us

$$
\sum_{j=0}^{k}\left(\begin{array}{l}
k \\
j
\end{array}\right)\left[\left(\ln \frac{b^{2}}{c}\right)^{k-j}+\left(\ln \frac{a^{2}}{c}\right)^{k-j}\right] E_{j}(a, b, c)=0
$$

Formula (3.5) follows.

The other formulas follow from Definition 2.1 and formula (3.2).

REMARK 3.3. For positive numbers $a, b$, and $c$, we have

$$
\begin{aligned}
& E_{0}(a, b, c)=1, \\
& E_{1}(a, b, c)=\ln c-\ln a-\ln b, \\
& E_{2}(a, b, c)=(\ln c-2 \ln a)(\ln c-2 \ln b), \\
& E_{3}(a, b, c)=\left[(\ln c-\ln a-\ln b)^{2}-3(\ln b-\ln a)^{2}\right](\ln c-\ln a-\ln b) .
\end{aligned}
$$

Since it is well known and easily established that the $E_{k}$ are integers, $E_{j}=0$ if $j$ is odd, and $E_{j}(0)=0$ if $j$ is positive and even, it follows from (3.3) and (3.2) that $E_{k}(a, b, c)$ is an integer polynomial in $\ln a, \ln b$, and $\ln c$ which is homogeneous of degree $k$ and which is divisible by $\ln c-\ln a-\ln b$ if $k$ is odd, and divisible by $(\ln c-2 \ln a)(\ln c-2 \ln b)$ if $k$ is even and positive. 
4. Some properties of the generalized Euler polynomials. In this section, we investigate properties of the generalized Euler polynomials defined by Definition 2.2.

THEOREM 4.1. For any given positive numbers $a, b$, and $c$ and $x \in \mathbb{R}$,

$$
\begin{aligned}
E_{k}(x ; a, b, c) & =\sum_{j=0}^{k}\left(\begin{array}{l}
k \\
j
\end{array}\right) \frac{(\ln c)^{k-j}}{2^{j}}\left(x-\frac{1}{2}\right)^{k-j} E_{j}(a, b, c), \\
E_{k}(x ; a, b, c) & =\sum_{j=0}^{k}\left(\begin{array}{l}
k \\
j
\end{array}\right)(\ln c)^{k-j}\left(\ln \frac{b}{a}\right)^{j}\left(x-\frac{1}{2}\right)^{k-j} E_{j}\left(\frac{\ln c-2 \ln a}{2(\ln b-\ln a)}\right), \\
E_{k}(x ; a, b, c) & =\sum_{j=0}^{k} \sum_{\ell=0}^{j}\left(\begin{array}{l}
k \\
j
\end{array}\right)\left(\begin{array}{l}
j \\
\ell
\end{array}\right) \frac{(\ln c)^{k-j}}{2^{j}}\left[\ln \frac{b}{a}\right]^{\ell}\left[\ln \frac{c}{a b}\right]^{j-\ell}\left[x-\frac{1}{2}\right]^{k-j} E_{\ell}, \\
E_{k}(a, b, c) & =2^{k} E_{k}\left(\frac{1}{2} ; a, b, c\right), \\
E_{k}(x) & =E_{k}(x ; 1, e, e) .
\end{aligned}
$$

Proof. By Definitions 2.1 and 2.2, we have

$$
\begin{aligned}
\frac{2 c^{2 x t}}{b^{2 t}+a^{2 t}} & =\sum_{k=0}^{\infty} 2^{k} E_{k}(x ; a, b, c) \frac{t^{k}}{k !} \\
\frac{2 c^{2 x t}}{b^{2 t}+a^{2 t}} & =\frac{2 c^{t}}{b^{2 t}+a^{2 t}} \cdot c^{(2 x-1) t} \\
& =\left(\sum_{k=0}^{\infty} \frac{t^{k}}{k !} E_{k}(a, b, c)\right)\left(\sum_{k=0}^{\infty} \frac{t^{k}}{k !}(2 x-1)^{k}(\ln c)^{k}\right) \\
& =\sum_{k=0}^{\infty}\left(\sum_{j=0}^{k}\left(\begin{array}{l}
k \\
j
\end{array}\right)(\ln c)^{k-j}(2 x-1)^{k-j} E_{j}(a, b, c)\right) \frac{t^{k}}{k !} .
\end{aligned}
$$

Equating the coefficients of $t^{k} / k$ ! in (4.6) yields

$$
2^{k} E_{k}(x ; a, b, c)=\sum_{j=0}^{k}\left(\begin{array}{l}
k \\
j
\end{array}\right)(\ln c)^{k-j}(2 x-1)^{k-j} E_{j}(a, b, c) .
$$

Formula (4.1) follows.

The other formulas follow directly from substituting formulas (3.2) and (3.3) into (4.1) and taking $x=1 / 2$ in (4.1), respectively. 
THEOREM 4.2. For positive integer $1 \leq p \leq k$,

$$
\begin{aligned}
\frac{\partial^{p}}{\partial x^{p}} E_{k}(x ; a, b, c) & =\frac{k !}{(k-p) !}(\ln c)^{p} E_{k-p}(x ; a, b, c), \\
\int_{\beta}^{x} E_{k}(t ; a, b, c) d t & =\frac{1}{(k+1) \ln c}\left[E_{k+1}(x ; a, b, c)-E_{k+1}(\beta ; a, b, c)\right] .
\end{aligned}
$$

PRoof. Differentiating equation (2.2) with respect to $x$ yields

$$
\frac{\partial}{\partial x} E_{k}(x ; a, b, c)=k(\ln c) E_{k-1}(x ; a, b, c) .
$$

Using formula (4.10) and by mathematical induction, formula (4.8) follows. Rearranging formula (4.10) produces

$$
E_{k}(x ; a, b, c)=\frac{1}{(k+1) \ln c} \frac{\partial}{\partial x} E_{k+1}(x ; a, b, c) .
$$

Formula (4.9) follows from integration on both sides of formula (4.11).

THEOREM 4.3. For positive numbers $a, b$, and $c$ and $x \in \mathbb{R}$,

$$
\begin{aligned}
E_{k}(x+1 ; a, b, c)= & \sum_{j=0}^{k}\left(\begin{array}{l}
k \\
j
\end{array}\right)(\ln c)^{k-j} E_{j}(x ; a, b, c), \\
E_{k}(x+1 ; a, b, c)= & 2 x^{k}(\ln c)^{k} \\
& +\sum_{j=0}^{k}\left(\begin{array}{l}
k \\
j
\end{array}\right)\left[(\ln c)^{k-j}-(\ln b)^{k-j}-(\ln a)^{k-j}\right] E_{j}(x ; a, b, c), \\
E_{k}(x+1 ; a, b, c)= & E_{k}\left(x ; \frac{a}{c}, \frac{b}{c}, c\right) .
\end{aligned}
$$

PROOF. From Definition 2.2 and straightforward calculation, we have

$$
\begin{aligned}
\frac{2 c^{x t}}{b^{t}+a^{t}} \cdot c^{t} & =\left[\sum_{k=0}^{\infty} \frac{t^{k}}{k !} E_{k}(x ; a, b, c)\right]\left[\sum_{k=0}^{\infty} \frac{t^{k}}{k !}(\ln c)^{k}\right] \\
& =\sum_{k=0}^{\infty}\left[\sum_{j=0}^{k}\left(\begin{array}{l}
k \\
j
\end{array}\right)(\ln c)^{k-j} E_{j}(x ; a, b, c)\right] \frac{t^{k}}{k !}, \\
\frac{2 c^{x t}}{b^{t}+a^{t}} \cdot c^{t} & =\frac{2 c^{(x+1) t}}{b^{t}+a^{t}}=\sum_{k=0}^{\infty} \frac{t^{k}}{k !} E_{k}(x+1 ; a, b, c) .
\end{aligned}
$$

Therefore, from equating the coefficients of $t^{k} / k$ ! in (4.15), formula (4.12) follows. 
Similarly, we obtain

$$
\begin{aligned}
\frac{2 c^{(x+1) t}}{b^{t}+a^{t}}= & \sum_{k=0}^{\infty} \frac{t^{k}}{k !} E_{k}(x+1 ; a, b, c)=2 c^{x t}+\frac{2 c^{x t}}{b^{t}+a^{t}}\left(c^{t}-b^{t}-a^{t}\right) \\
= & 2 \sum_{k=0}^{\infty} \frac{t^{k}}{k !} x^{k}(\ln c)^{k} \\
& +\left[\sum_{k=0}^{\infty} \frac{t^{k}}{k !} E_{k}(x ; a, b, c)\right]\left[\sum_{k=0}^{\infty}\left((\ln c)^{k}-(\ln b)^{k}-(\ln a)^{k}\right) \frac{t^{k}}{k !}\right] \\
= & \sum_{k=0}^{\infty}\left[2 x^{k}(\ln c)^{k}\right. \\
& \left.\quad+\sum_{j=0}^{k}\left(\begin{array}{l}
k \\
j
\end{array}\right)\left[(\ln c)^{k-j}-(\ln b)^{k-j}-(\ln a)^{k-j}\right] E_{j}(x ; a, b, c)\right] \frac{t^{k}}{k !} .
\end{aligned}
$$

By equating coefficients of $t^{k} / k$ !, we obtain formula (4.13).

Since

$$
\begin{aligned}
\sum_{k=0}^{\infty} \frac{t^{k}}{k !} E_{k}(x+1 ; a, b, c) & =\frac{2 c^{(x+1) t}}{b^{t}+a^{t}}=\frac{2 c^{x t}}{(b / c)^{t}+(a / c)^{t}} \\
& =\sum_{k=0}^{\infty} \frac{t^{k}}{k !} E_{k}\left(x ; \frac{a}{c}, \frac{b}{c}, c\right)
\end{aligned}
$$

by equating coefficients, we obtain formula (4.14). The proof is complete.

COROLLARY 4.4. The following formulas are valid for positive numbers $a$, $b$, and $c$ and real number $x$ :

$$
\begin{aligned}
& E_{k}(x+1)+E_{k}(x)=2 x^{k}, \\
& E_{k}(x+1)=\sum_{j=0}^{k}\left(\begin{array}{l}
k \\
j
\end{array}\right) E_{j}(x), \\
& E_{k}(x+1 ; 1, b, b)+E_{k}(x ; 1, b, b)=2 x^{k}(\ln b)^{k}, \\
& E_{k}(x+1 ; 1, b, b)=\sum_{j=0}^{k}\left(\begin{array}{l}
k \\
j
\end{array}\right) E_{j}(x ; 1, b, b)(\ln b)^{k-j}, \\
& \sum_{j=0}^{k-1}\left(\begin{array}{c}
k \\
j
\end{array}\right) E_{j}(x ; 1, b, b)(\ln b)^{k-j}+2 E_{k}(x ; 1, b, b)=2 x^{k}(\ln b)^{k}, \\
& \int_{x}^{x+1} E_{k}(t ; a, b, c) d t=\frac{1}{(k+1) \ln c} \sum_{j=0}^{k}\left(\begin{array}{c}
k+1 \\
j
\end{array}\right)(\ln c)^{k-j} E_{j}(x ; a, b, c) .
\end{aligned}
$$


THEOREM 4.5. For positive numbers $a, b, c>0, x \in \mathbb{R}$, and nonnegative integer $k$,

$$
\begin{aligned}
& E_{k}(1-x ; a, b, c)=(-1)^{k} E_{k}\left(x ; \frac{c}{a}, \frac{c}{b}, c\right), \\
& E_{k}(1-x ; a, b, c)=E_{k}\left(-x ; \frac{a}{c}, \frac{b}{c}, c\right) .
\end{aligned}
$$

Proof. From Definition 2.2 and easy computation, we have

$$
\begin{aligned}
\sum_{k=0}^{\infty} \frac{t^{k}}{k !} E_{k}(1-x ; a, b, c) & =\frac{2 c^{(1-x) t}}{b^{t}+a^{t}}=\frac{2 c^{t} \cdot c^{-x t}}{b^{t}+a^{t}}=\frac{2 c^{-x t}}{(c / b)^{-t}+(c / a)^{-t}} \\
& =\sum_{k=0}^{\infty} \frac{t^{k}}{k !}(-1)^{k} E_{k}\left(x ; \frac{c}{a}, \frac{c}{b}, c\right) .
\end{aligned}
$$

Equating coefficients of $t^{k}$ above leads to formula (4.24).

By the same procedure, we can establish formula (4.25).

THEOREM 4.6. For positive numbers $a, b, c>0$, nonnegative natural number $k$, and $x, y \in \mathbb{R}$,

$$
\begin{aligned}
& E_{k}(x+y ; a, b, c)=\sum_{j=0}^{k}\left(\begin{array}{l}
k \\
j
\end{array}\right)(\ln c)^{k-j} y^{k-j} E_{j}(x ; a, b, c), \\
& E_{k}(x+y ; a, b, c)=\sum_{j=0}^{k}\left(\begin{array}{l}
k \\
j
\end{array}\right)(\ln c)^{k-j} x^{k-j} E_{j}(y ; a, b, c) .
\end{aligned}
$$

Proof. These two formulas can be deduced from the following calculation and considering symmetry of $x$ and $y$ :

$$
\begin{aligned}
\sum_{k=0}^{\infty} \frac{t^{k}}{k !} E_{k}(x+y ; a, b, c) & =\frac{2 c^{(x+y) t}}{b^{t}+a^{t}}=\frac{2 c^{x t} \cdot c^{y t}}{b^{t}+a^{t}} \\
& =\left[\sum_{k=0}^{\infty} \frac{t^{k}}{k !} E_{k}(x ; a, b, c)\right]\left[\sum_{k=0}^{\infty} \frac{t^{k}}{k !}(\ln c)^{k} y^{k}\right] \\
& =\sum_{k=0}^{\infty}\left[\sum_{j=0}^{k}\left(\begin{array}{l}
k \\
j
\end{array}\right)(\ln c)^{k-j} y^{k-j} E_{j}(x ; a, b, c)\right] \frac{t^{k}}{k !} .
\end{aligned}
$$

The proof is complete.

THEOREM 4.7. For natural numbers $k$ and $m$ and positive number $b$,

$$
\sum_{\ell=1}^{m}(-1)^{\ell} \ell^{k}=\frac{1}{2(\ln b)^{k}}\left[(-1)^{m} E_{k}(m+1 ; 1, b, b)-E_{k}(1 ; 1, b, b)\right] .
$$


Proof. Rearranging formula (4.20) gives us

$$
x^{k}=\frac{1}{2(\ln b)^{k}}\left[E_{k}(x+1 ; 1, b, b)+E_{k}(x ; 1, b, b)\right] .
$$

Replacing $x$ by $\ell \in \mathbb{N}$ and summing up $\ell$ from 1 to $m$ yields

$$
\begin{aligned}
\sum_{\ell=1}^{m}(-1)^{\ell} \ell^{k} & =\frac{1}{2(\ln b)^{k}} \sum_{\ell=1}^{m}(-1)^{\ell}\left[E_{k}(\ell+1 ; 1, b, b)+E_{k}(\ell ; 1, b, b)\right] \\
& =\frac{1}{2(\ln b)^{k}}\left[(-1)^{m} E_{k}(m+1 ; 1, b, b)-E_{k}(1 ; 1, b, b)\right] .
\end{aligned}
$$

The proof is complete.

REMARK 4.8. Finally, we give several concrete formulas as follows:

$$
\begin{aligned}
E_{0}(x ; a, b, c)= & 1 \\
E_{1}(x ; a, b, c)= & \left(x-\frac{1}{2}\right) \ln c+\frac{1}{2}(\ln c-\ln a-\ln b) \\
E_{2}(x ; a, b, c)= & \left(x-\frac{1}{2}\right)^{2}(\ln c)^{2}+\left(x-\frac{1}{2}\right)(\ln c-\ln b-\ln a) \ln c \\
& +\frac{1}{4}(\ln c-2 \ln a)(\ln c-2 \ln b) .
\end{aligned}
$$

ACKNOWLEDGments. The authors would like to express many thanks to the anonymous referees for their valuable comments. The first two authors were supported in part by NNSF of China, Grant 10001016, SF for the Prominent Youth of Henan Province, Grant 0112000200, SF of Henan Innovation Talents at Universities, NSF of Henan Province, Grant 004051800, Doctor Fund of Jiaozuo Institute of Technology, China. The third author was partially supported by a grant of the Faculty Research Council of the University of Texas-Pan American.

\section{REFERENCES}

[1] M. Abramowitz and I. A. Stegun (eds.), Handbook of Mathematical Functions, with Formulas, Graphs, and Mathematical Tables, 3rd ed., with corrections, National Bureau of Standards, Applied Mathematics Series, vol. 55, US Government Printing Office, Washington, D.C., 1965.

[2] B.-N. Guo and F. Qi, Generalization of Bernoulli polynomials, Internat. J. Math. Ed. Sci. Tech. 33 (2002), no. 3, 428-431.

[3] Q.-M. Luo, B.-N. Guo, F. Qi, and L. Debnath, Generalizations of Bernoulli numbers and polynomials, Int. J. Math. Math. Sci. 2003 (2003), no. 59, 3769-3776.

[4] Q.-M. Luo and F. Qi, Relationships between generalized Bernoulli numbers and polynomials and generalized Euler numbers and polynomials, Adv. Stud. Contemp. Math. (Kyungshang) 7 (2003), no. 1, 11-18, RGMIA Res. Rep. Coll. 5 (2002), no. 3, Art. 1, 405-412, http://rgmia.vu.edu.au/v5n3.html.

[5] F. Qi and B.-N. Guo, Generalisation of Bernoulli polynomials, RGMIA Res. Rep. Coll. 4 (2001), no. 4, Art. 10, 691-695, http://rgmia.vu.edu.au/v4n4.html. 
[6] Zh.-X. Wang and D.-R. Guo, Introduction to Special Function, The Series of Advanced Physics of Peking University, Peking University Press, Beijing, 2000 (Chinese).

Qiu-Ming Luo: Department of Broadcast-Television Teaching, Jiaozuo University, Jiaozuo City, Henan 454002, China

E-mail address: 1uoqm@jzu.edu.cn

Feng Qi: Department of Applied Mathematics and Informatics, Jiaozuo Institute of Technology, Jiaozuo City, Henan 454000, China

E-mail address: qi feng@jzit.edu.cn

URL: http://rgmia.vu.edu.au/qi .htm1

Lokenath Debnath: Department of Mathematics, University of Texas-Pan American, Edinburg, TX 78539, USA

E-mail address: debnath1@panam.edu 


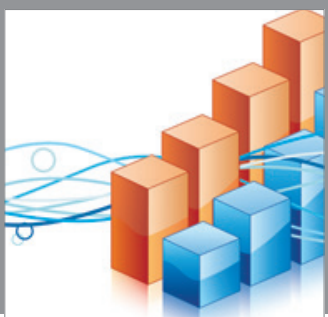

Advances in

Operations Research

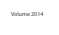

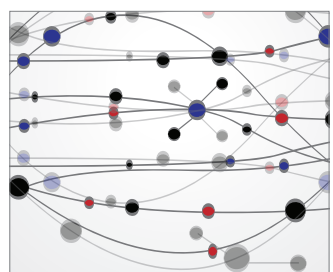

\section{The Scientific} World Journal
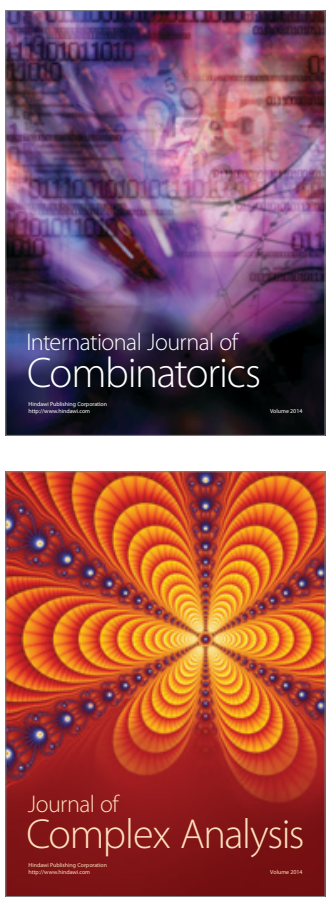

International Journal of

Mathematics and

Mathematical

Sciences
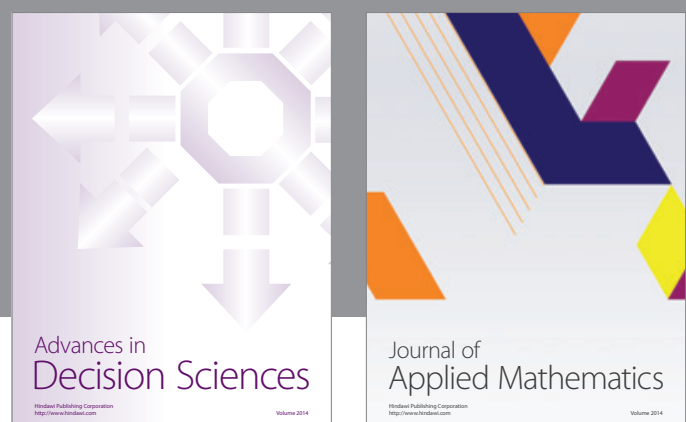

Journal of

Applied Mathematics
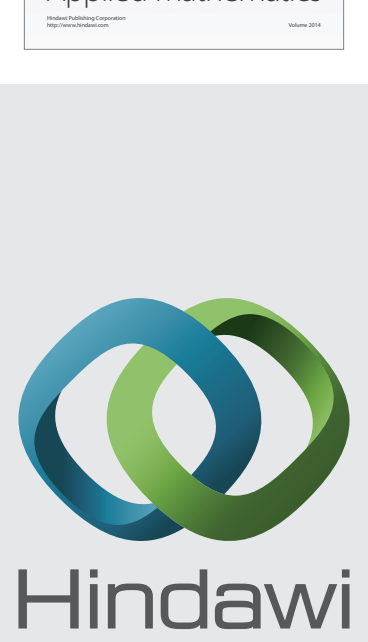

Submit your manuscripts at http://www.hindawi.com
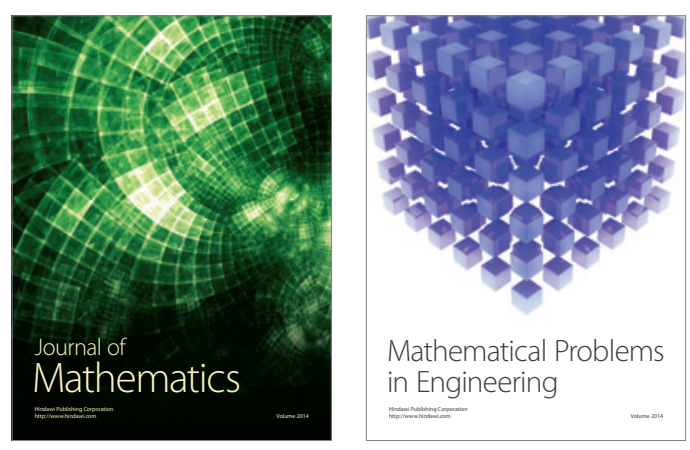

Mathematical Problems in Engineering
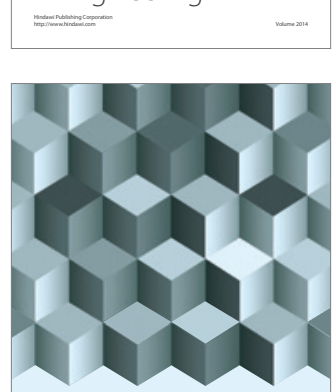

Journal of

Function Spaces
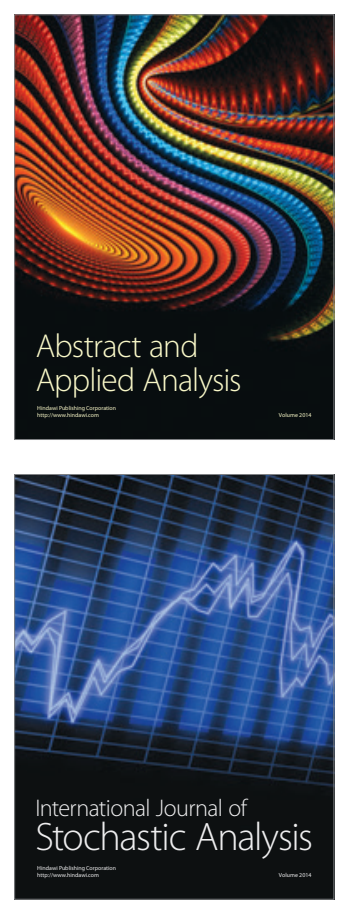

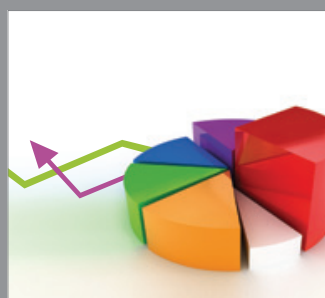

ournal of

Probability and Statistics

Promensencen
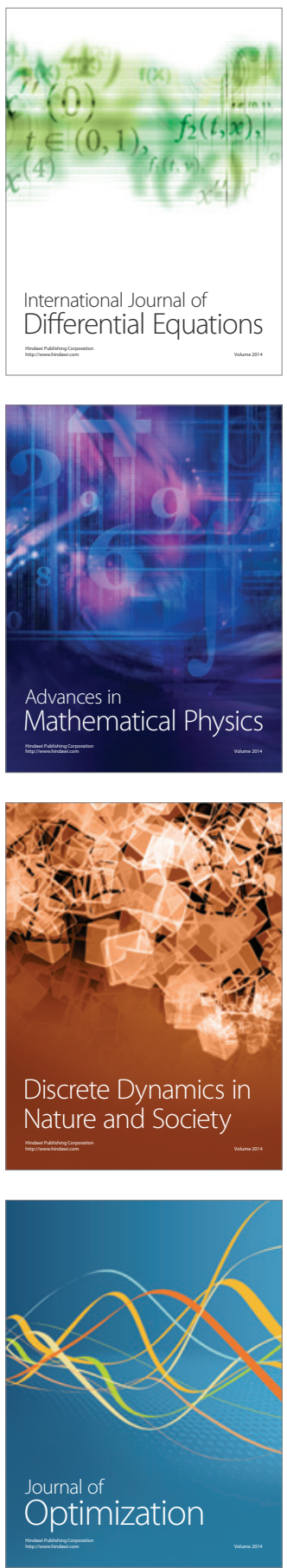